\title{
Polarization properties of dipolelike defect modes in photonic crystal nanocavities
}

\author{
Oskar Painter and Kartik Srinivasan \\ Department of Applied Physics, California Institute of Technology, Pasadena, California 91125
}

Received July 20, 2001

\begin{abstract}
Far-field measurements of the in-plane polarization properties of spontaneous emission from optical nanocavities formed in two-dimensional photonic crystal slab waveguides are presented. A strong polarization signature, even subthreshold, is found for a pair of highly localized dipolelike resonant modes. This polarization signature is used to study the effects of symmetry lowering within the cavity. (C) 2002 Optical Society of America

OCIS codes: $140.5960,230.5750$.
\end{abstract}

Optical nanocavities formed in two-dimensional (2D) photonic crystal (PC) slab waveguides ${ }^{1,2}$ can be designed to support resonant modes with modal volumes close to the theoretical limit of a cubic half-wavelength. ${ }^{3,4}$ The ability to define the cavity geometry through the use of planar fabrication techniques provides a great deal of flexibility in the design of cavity modes, their wavelength, polarization, and emission patterns. Such flexibility, which is important for nano-optic devices capable of high-density integration within light-wave circuits, has already been used to create a multiwavelength 2D laser array ${ }^{5}$ and resonant cavities capable of supporting both highly localized donor- and acceptor-type modes. ${ }^{6}$ Significant progress has also been made using similar planar PC waveguide structures to form in-plane waveguides, ${ }^{7-9}$ coupled-resonator waveguide systems, ${ }^{10,11}$ and resonant add-drop devices. ${ }^{12}$

In this Letter we study the polarization properties of a pair of highly localized deep donor (DD) type modes, termed $x$ and $y$ dipolelike modes, as they transform as vector components within the plane of the slab waveguide. $^{3} \quad$ The PC defect cavities studied here are realized in an optically thin dielectric slab patterned with a 2D hexagonal array of air holes ${ }^{13}$ [Fig. 1(a)]. As a result of the localized nature of the defect region and the symmetry of the PC in which it is embedded, the field patterns of the DD modes resemble that of an oscillating electric dipole in the plane of the waveguide [Figs. 2(a) and 2(b)]. For defect cavities in which the PC lattice is relatively porous, as is the case here, radiation from the dipolelike modes is predominantly $(\geq 90 \%)$ emitted out of plane from the patterned slab waveguide. A plot based upon finite-difference time-domain (FDTD) simulations of collection efficiency versus collection angle for the vertically emitted power from the $x$ dipole mode is given in Fig. 2(c). FDTD calculations show that $\geq 99 \%$ of this vertically emitted power is carried in the dominant ( $E_{x}$ or $E_{y}$ ) polarization of the ( $x$ or $y$ ) dipolelike modes.

In defect cavities that maintain the sixfold symmetry $\left[C_{6 v}\right.$ (Ref. 3)] of the host hexagonal lattice, the DD dipolelike modes are degenerate, and any mixture of the $x$ and $y$ dipolelike modes shown in Fig. 2 may be excited. To break this degeneracy and to resolve the polarized $x$ and $y$ dipolelike modes, the cavity symmetry must be reduced to $C_{2 v}$. To this end, three different cavity geometries, shown in Figs. 1(b)-1(d), are created. The symmetric (S) cavity, created by the complete removal of a central hole, eliminates translational symmetries while maintaining the point group symmetry of the lattice, $C_{6 v}$. The X- and Y-split cavities represent slight modifications of the $\mathrm{S}$ cavity that reduce the symmetry of the system from $C_{6 v}$ to $C_{2 v} \cdot{ }^{3}$ In the $\mathrm{X}$-split cavity, air holes on the top and at the bottom of the central missing hole are moved inward a distance

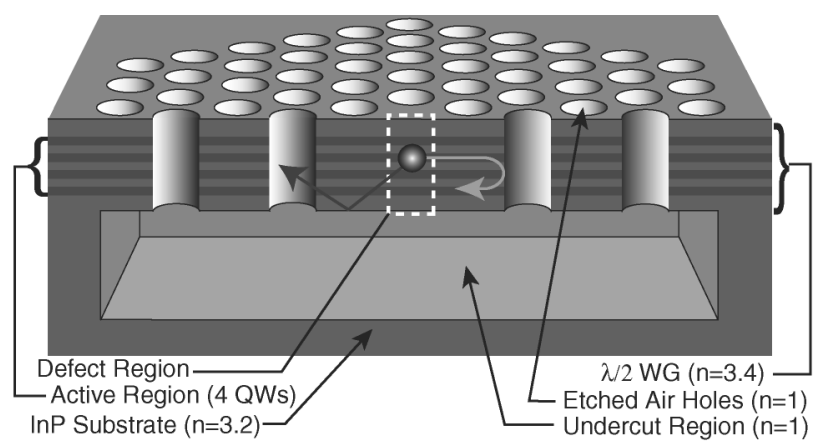

(a)

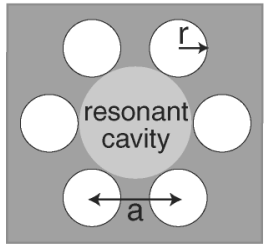

(b)

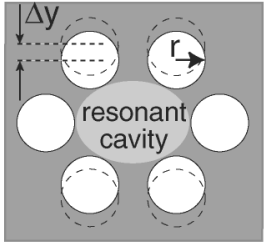

(c)

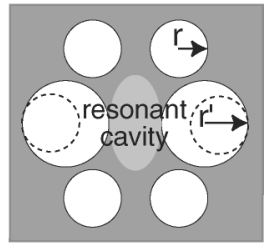

(d)
Fig. 1. (a) Cross section of a defect cavity in a $2 \mathrm{D} \mathrm{PC}$ slab waveguide (WG). QWs, quantum wells. (b)-(d) PC cavity geometries with different symmetries. For operation at $\lambda=1.5 \mu \mathrm{m}$, the slab thickness, hole radius, and lattice spacing are roughly $d=200 \mathrm{~nm}, r=175 \mathrm{~nm}$, and $a=500 \mathrm{~nm}$, respectively. 


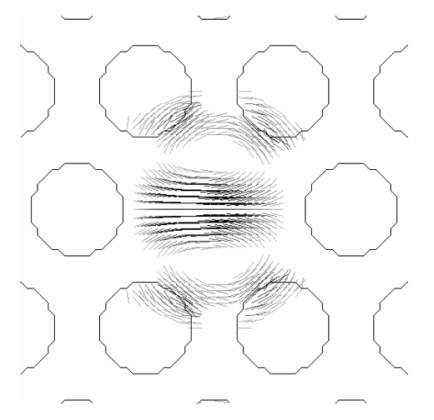

(a)

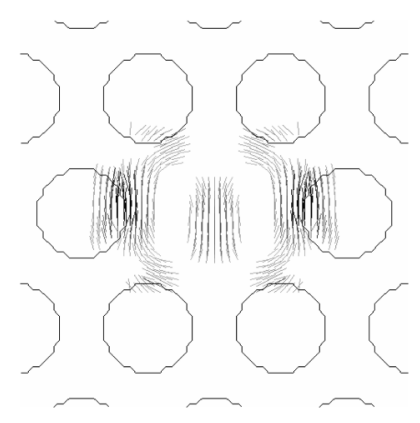

(b)

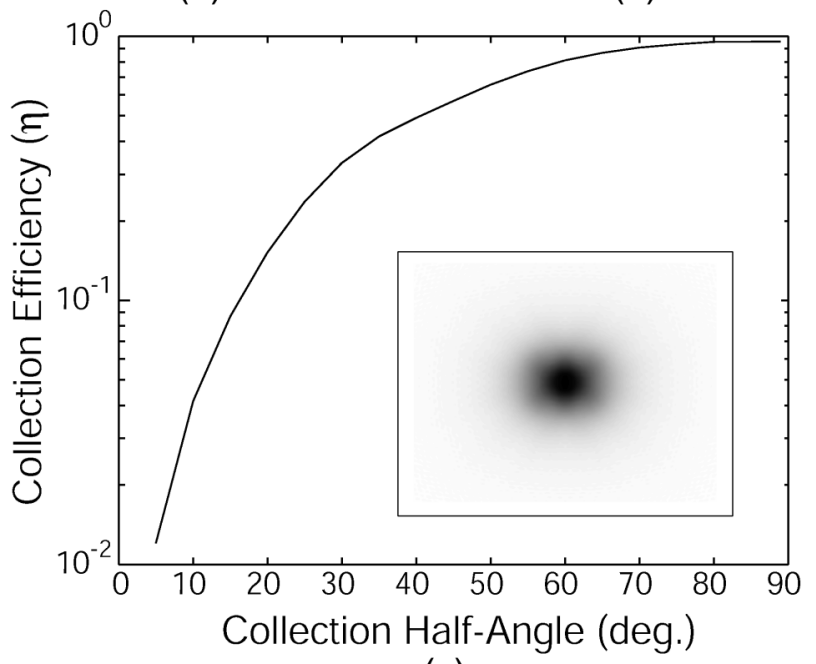

(c)

Fig. 2. (a), (b) FDTD-generated vector field patterns of the electric field within the symmetry plane of the slab waveguide for the $x$ and $y$ dipolelike modes. (c) Collection efficiency of the vertically emitted power versus collection half-angle (plot of the $E_{x}$ intensity in the far field for the $x$ dipolelike mode shown in the inset). FDTD simulations were performed with $n_{\text {slab }}=3.4, r / a=0.30$, and $d / a=0.55$.

$\Delta y$, and in the Y-split cavity, the missing central hole is accompanied on either side by an enlarged hole of radius $r^{\prime}>r$.

Spectroscopy of the resonant mode structure of the defect cavities was performed by analysis of the light emission in the 1500-nm band from an integral InGaAsP/InP multiple-quantum-well active region grown within the dielectric slab waveguide. ${ }^{13} \quad$ A large array of defect cavities was fabricated with varying lattice spacings and hole sizes so that a wide range of resonant mode wavelengths would be covered. The defect cavities were fabricated in sets of three ( $\mathrm{S}$, $\mathrm{X}, \mathrm{Y}$ ), with each of the different cavities within the triplet designed to have the same lattice spacing and nominal hole radius. A lattice spacing of approximately $550 \mathrm{~nm}(r / a=0.35, d / a=0.41)$ was found to provide the best overlap of the DD modes of all three cavities and the quantum-well emission band. The defect cavities were fabricated with eight periods of the $\mathrm{PC}$ surrounding the central defect region, limiting the leakage of light in plane and allowing for efficient light collection from the top of the sample. ${ }^{3}$

The sample was mounted on an $x-y-z$ stage, and the defect cavities were optically pumped by an 830-nm semiconductor laser diode at an angle normal to the sample surface (Fig. 3). A high-numerical-aperture (N.A., 0.5), long-working-distance, $100 \times$ objective lens was used to image the defect cavities, collect the photoluminescence (PL), and focus the optical pump beam. A GaAs filter was used to separate the pump beam from the PL before detection. The spot size of the pump beam on the sample surface was approximately $4 \mu \mathrm{m}$, covering half the diameter of a PC cavity. Pulsed pumping conditions (10-ns pulses with $1 \%$ duty cycle) were used to reduce heating of the membrane structure. Pump power was maintained below the lasing threshold of any of the cavity modes for all measurements. The fraction of the total vertically emitted power from the dipolelike modes collected within the $30^{\circ}$ half-angle of the objective lens is estimated to be $30 \%$ from FDTD calculations [Fig. 2(c)].

The polarization of the collected PL was measured with a polarizer placed in the beam path after the collection lens. In this position, the polarizer filters the different in-plane polarization components of the emitted light from the PC slab waveguide. For each of the cavities studied, three PL spectra were taken: one with the polarizer removed (NP), one with the polarizer oriented along PX, and one with the polarizer oriented along PY. The orthogonal PX and PY angles of the polarizer, shown projected onto the surface of a defect cavity in the inset of Fig. 3, were chosen to correspond to the orientation of the $x$ and $y$ dipolelike modes, respectively. For reference, a PL spectrum from an unprocessed region on the sample is shown in Fig. 4(a). The two peaks in the spectra correspond to the heavy-hole-to-conduction band (hh-c) and light-hole-to-conduction band (lh-c) transitions in the quantum well. ${ }^{13}$

The PL from a S cavity is shown in Fig. 4(b). With the polarizer removed from beam path (NP), a single resonance peak near $1550 \mathrm{~nm}$ is present. The normalized frequency $\left(a / \lambda_{0}\right)$ of this peak is 0.355 , which lies near the middle of the TE-like guided-mode

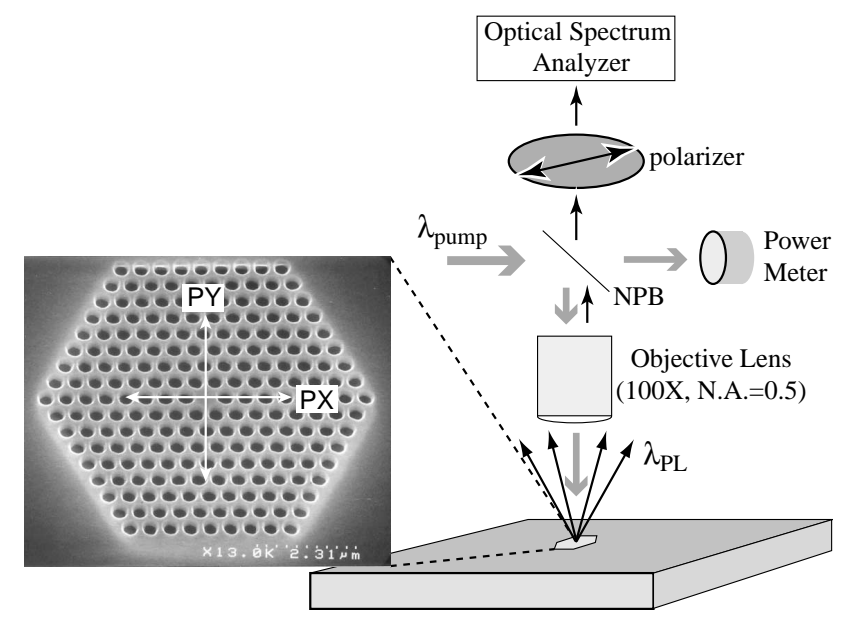

Fig. 3. Schematic of the polarization-selective PL measurement setup. The inset shows a projection of the PX and PY polarizer orientations onto a scanning electron microscope image of a fabricated defect cavity as it would be mounted in the PL setup. NBP, nonpolarizing beam splitter. 


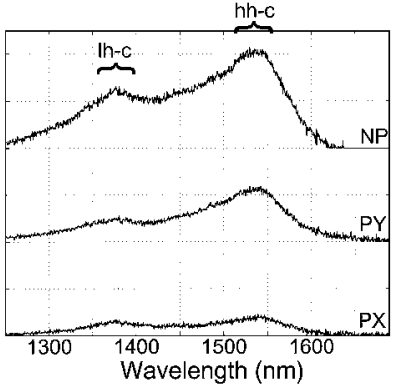

(a)

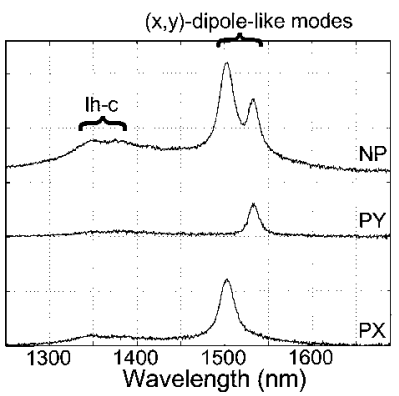

(c)

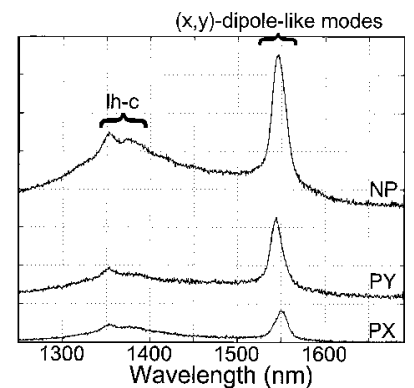

(b)

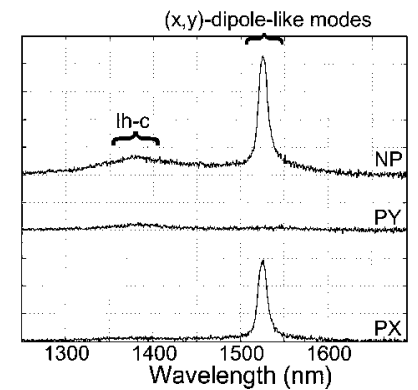

(d)
Fig. 4. Polarization-filtered PL from the (a) unprocessed area of the sample (no cavity), (b) S cavity, (c) X-split cavity, and (d) Y-split cavity.

bandgap and corresponds well to the predicted frequency of the dipolelike modes from FDTD simulations. This resonance peak contains energy in both the PX and the PY polarizations. The two polarization peaks are nearly degenerate, consistent with the degeneracy of the $(x, y)$ dipolelike modes in a cavity with $C_{6 v}$ symmetry. An observed 5-nm splitting of the polarized PL peaks arises from asymmetries in the defect cavity that are unintentionally introduced during fabrication.

In Fig. 4(c), the PL spectrum from an X-split cavity with $\Delta y=25 \mathrm{~nm}$ is shown. The single resonant peak of the $\mathrm{S}$ cavity has now split into two distinct peaks separated in wavelength by $30 \mathrm{~nm}$. The two peaks are to be orthogonally polarized, the long-wavelength peak along PY and the short-wavelength peak along PX. Referring to the vector plots of Fig. 2, the long-wavelength peak is the $y$ dipole mode and the short-wavelength peak is the $x$ dipole mode. This classification is further supported by FDTD simulations, which predict that the $x$ dipole mode will have a shorter wavelength than the $y$ dipole mode in an X-split cavity. ${ }^{6}$

PL from a Y-split cavity with $r^{\prime} / a=0.5$ is shown in Fig. 4(d). A single resonance peak, polarized along PX, remains. Energy in the orthogonal PY polarization, which was present in both the S and X-split cavities, is now absent. This result is consistent with FDTD simulations, which show that the $y$ dipole mode is strongly perturbed for $r^{\prime} / a>0.45$, leaving only the $x$ dipole mode in the guided-mode bandgap. ${ }^{3}$ The measured peak power of the $x$ dipole peak varies sinusoidally with polarizer angle, with a peak-to-valley ratio of approximately 25:1. This value is lower than the theoretically estimated polarization extinction ratio of the $x$ dipole, most likely as a result of the collection of nonresonant emission along with the $x$ dipole mode radiation.

In summary, the simple picture of the DD modes as a pair of oscillating dipole sources, evoked by the vector plots of Fig. 2, carries over quite well to the far-field polarization measurements. Adjustments in the defect-cavity symmetry result in splitting of the dipole-mode degeneracy, as predicted by group theory. Once they are split in frequency, the $x$ and $y$ dipolelike modes are strongly polarized along two different orthogonal directions, consistent with their near-field dipole character. A polarization extinction ratio of at least 25:1 is found for the $x$ dipole mode of the Y-split cavity. Apart from technological applications, the polarization signature of the dipolelike modes is extremely valuable in understanding the spectra of more-complex defect cavities and in estimating the accuracy of numerical models such as the FDTD model.

The authors thank A. Scherer, P. D. Dapkus, and J. D. O'Brien for their help with the growth and fabrication of samples. K. Srinivasan thanks the Hertz Foundation for its financial support. O. Painter's e-mail address is opainter@its.caltech.edu.

\section{References}

1. P. St. J. Russell, D. M. Atkin, and T. A. Birks, Bound Modes of Two-Dimensional Photonic Crystal Waveguides (Kluwer Academic, Dordrecht, The Netherlands, 1996), pp. 203-218.

2. S. G. Johnson, S. Fan, P. R. Villeneuve, J. D. Joannopoulos, and L. A. Kolodziejaki, Phys. Rev. B 60, 5751 (1999).

3. O. Painter, J. Vučković, and A. Scherer, J. Opt. Soc. Am. B 16, 275 (1999).

4. R. Coccioli, M. Boroditsky, K. W. Kim, Y. RahmatSamii, and E. Yablonovitch, IEE Proc. Optoelectron. 145, 391 (1996).

5. O. Painter, A. Husain, A. Scherer, P. T. Lee, I. Kim, J. D. O'Brien, and P. D. Dapkus, IEEE Photon. Technol. Lett. 12, 1126 (2000).

6. O. Painter, K. Srinivasan, J. D. O’Brien, A. Scherer, and P. D. Dapkus, J. Opt. A 3, S161 (2001).

7. E. Chow, S. Y. Lin, J. R. Wendt, S. G. Johnson, and J. D. Joannopoulos, Opt. Lett. 26, 286 (2001).

8. T. Baba, N. Fukaya, and J. Yonekura, Electron. Lett. 35, 654 (1999).

9. M. Lončar, D. Nedeljković, T. Doll, J. Vučković, A. Scherer, and T. P. Pearsall, Appl. Phys. Lett. 77, 1937 (2000).

10. C. Smith, R. De la Rue, M. Rattier, S. Olivier, H. Benisty, C. Weisbuch, T. Krauss, U. Oesterlé, and R. Houdré, Appl. Phys. Lett. 78, 1487 (2001).

11. S. Olivier, C. Smith, M. Rattier, H. Benisty, C. Weisbuch, T. Krauss, R. Houdré, and U. Oesterlé, Opt. Lett. 26, 1019 (2001).

12. S. Noda, A. Chutinan, and M. Imada, Nature 407, 608 (2000).

13. O. J. Painter, A. Husain, A. Scherer, J. D. O’Brien, I. Kim, and P. D. Dapkus, J. Lightwave Technol. 17, 2082 (1999). 